\section{Response to Spencer and Graham Article}

\section{The Mathematical Intelligencer encourages comments}

about the material in this issue. Letters to the editor

should be sent to either of the editors-in-chief, Chandler

Davis or Marjorie Senechal.

$\mathrm{n}$ a recent issue, $\mathrm{J}$. Spencer and $\mathrm{R}$. Graham wrote an article ("The Elementary Proof of the Prime Number Theorem", Mathematical Intelligencer vol. 31 (2009), no. 1, 18-23) which presented a posthumous note by E. G. Straus.

In the article, they also include excerpts from a lengthy interview we had with Atle Selberg; parts of this appeared in N. A. Baas and C. F. Skau, "The Lord of the Numbers, Atle Selberg. On his Life and Mathematics", Bull. Amer. Math. Soc. (2008), 617-649. In order to understand Selberg's point of view, we think it is important to read his complete account in the interview. This is available at www.math.ntnu.no/ Selberg-interview/PNT/. There, one finds Selberg's complete statement-some of it was left out in the Bulletin article, including a letter and report from Hermann Weyl to which Selberg refers. We think that this material, especially the Weyl report, is of great historical interest, and we recommend it to the reader. Hermann Weyl's role in all this becomes clearer and more balanced than in Straus's somewhat biased view.

The reader is also referred to the interesting article by D. Goldfeld, "The Elementary Proof of the Prime Number Theorem: An Historical Perspective" (in Number Theory: New York Seminar 2003, Eds. D. Chudnovsky, G. Chudnovsky and M. Nathanson, New York: Springer, 179-192) (www.math.columbia.edu/ goldfeld/ErdosSelbergDispute.pdf).
Here, Selberg refutes in his correspondence with Goldfeld several of the claims that E. G. Straus makes, in particular, the claim that he, Selberg, did not appropriately refer to Erdös in his published paper.

In the interview, Selberg also openly explains how he tried to get Erdós off the track. In the excerpt (see the webpage above) from our interview at the bottom of page 9, Selberg says: "I kind of tried to scare him away from the prime number theorem itself. It was, one may say, a little dishonest that I did not tell him that my counterexample was based on a nonmonotonic function." Furthermore, in the interview Selberg states that his goal was to prove the PNT by using his fundamental formula; see the excerpt page 5 , lower part.

Goldfeld writes in his article on page 8 (lower part), quoting a letter from Selberg: "This attempt to throw Erdo"s off the track (clearly not succeeding!) is somewhat understandable given my mood at the time."

It is our impression that Selberg wanted to work towards the prime number theorem at his own pace using his fundamental formula, and in his attempt to lead Erdös away from it, he apparently gave Erdös the impression that he thought this would not lead to a proof. This seems to have caused much of the controversy.

We think that this additional information provides a better picture of the circumstances around the elementary proof of the prime number theorem and should be of great interest for the mathematical community.

Nils A. Baas

Department of Mathematical Sciences

Norwegian University of Science and Technology

NO-7491 Trondheim

Norway

e-mail: baas@math.ntnu.no

Christian F. Skau

Department of Mathematical Sciences

Norwegian University of Science and Technology

NO-7491 Trondheim

Norway

e-mail: csk@math.ntnu.no 